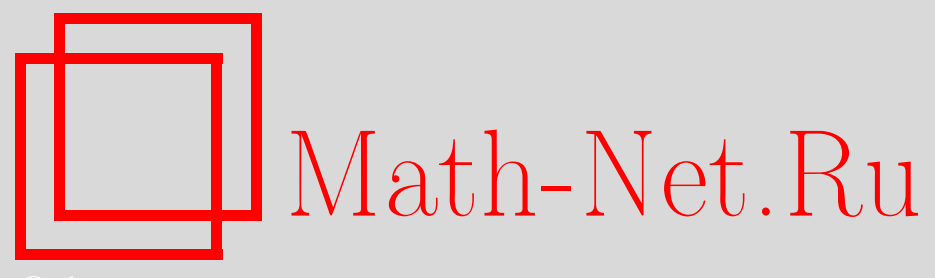

Л. Р. Мутафчиев, Предельная теорема о форме графов Ферре, Дискрет. матем., 1999, том 11, выпуск 1, 76-96

DOI: https://doi.org/10.4213/dm365

Использование Общероссийского математического портала Math-Net.Ru подразумевает, что вы прочитали и согласны с пользовательским соглашением http://www . mathnet.ru/rus/agreement

Параметры загрузки:

IP: 54.157 .27 .8

26 апреля 2023 г., 11:10:34 


\section{Предельная теорема о форме графов Ферре}

(C) 1999 г. Л. Р. Мутафчиев

Изучается асимптотическое поведение $s$-й по величине части $L_{s, n}$ случайного разбиения положительного целого числа $n$ при $n \rightarrow \infty$. Доказана слабая сходимость распределения $L_{s, n}$ к нормальному распределению, когда $s$ имеет порядок $n^{1 / 2}$.

Работа выполнена при поддержке Министерства образования, науки и техники Болгарии, контракт 705/97. Часть работы выполнена во время пребывания автора в Математическом институте им. В. А. Стеклова РАН.

\section{1. Введение}

Разбиение положительного целого числа $n$ ( $n$-разбиение) - это представление его в виде суммы положительных целых чисел без учета порядка слагаемых, эти слагаемые называются частями разбиения. Асимптотика числа $n$-разбиений, обычно обозначаемого $p(n)$, была получена Харди и Раманужданом [8]. Асимптотическое разложение, позволяющее вычислять $p(n)$ с большой точностью, было получено позднее Радемахером (см., например, гл. 5 в [2]). Зададим равномерное распределение вероятностей $\mathbf{P}$ на множестве всех $n$-разбиений, полагая, что каждому $n$-разбиению приписана одинаковая вероятность $1 / p(n)$. Символы $\mathbf{E}$ и $\mathbf{D}$ обозначают математическое ожидание и дисперсию относительно распределения Р. Таким образом, любая числовая характеристика разбиения является случайной величиной. Эрдёш и Лехнер [6], были, по-видимому, первыми, кто изучал случайные разбиения, используя описанный выше вероятностный подход. Чтобы описать полученные ими результаты, обозначим $Z_{s, n}$ число частей, больших $s-1$ в случайном $n$-разбиении, $s \geqslant 1$. Очевидно, что $Z_{1, n}$ равно общему числу частей в случайном разбиении. Эрдёш и Лехнер в [8] доказали, что при $n \rightarrow \infty$

$$
\mathrm{E} Z_{1, n} \sim \pi^{-1}(3 / 2)^{1 / 2} n^{1 / 2} \log n
$$

и, более того, что при подходящей нормировке $Z_{1, n}$ имеет нетривиальное предельное распределение, именно, для любого фиксированного $v,-\infty<v<\infty$,

$$
\lim _{n \rightarrow \infty} \mathbf{P}\left\{\frac{\pi Z_{1, n}}{\sqrt{6 n}}-\log \frac{\sqrt{6 n}}{\pi}<v\right\}=e^{-e^{-v}} .
$$

Следует отмеить, что в силу свойства двойственности разбиений, наглядно представляемого с помощью графов Ферре, то же предельное распределение имеет и наибольшая часть случайного разбиения, которое в дальнейшем будет обозначаться 
$L_{1, n}$. Вообще, пусть $L_{k, n}$ обозначает $k$-ю по величине часть случайного $n$-разбиения. Граф Ферре с $n$ точками определяется как плоская диаграмма, состоящая из столбцов, составленных из равноотстоящих точек так, что нижние точки столбцов расположены на одном горизонтальном уровне, общее число точек равно $n$ и высота $k$-го столбца равна $L_{k, n}$. Числа точек в строках этой диаграммы, начиная с нижней строки, образуют сопряженную к $\left\{L_{k, n}\right\}$ диаграмму (разбиение) $\left\{L_{k, n}^{*}\right\}$. Так как $\left\{L_{k, n}\right\}$ равномерно распределены на множестве всех диаграмм размера $n$, сопряженные диаграммы $\left\{L_{k, n}^{*}\right\}$ также равномерно распределены. Пусть $X_{j, n}$.обозначает число частей, равных $j$, в случайном $n$-разбиении, $j=1, \ldots, n$. Заметим, что существует простая связь между $\left\{L_{k, n}^{*}\right\}$ и суммами величин $X_{j, n}$, именно,

$$
L_{k, n}^{*}=\sum_{j \geqslant k} X_{j, n}=Z_{k, n}
$$

при $k \geqslant 1$. Отсюда следует, что $L_{k, n}$ и $Z_{k, n}$ имеют одинаковое распределение вероятностей, и в силу этой двойственности результаты о распределении одной из величин $Z_{k, n}$ и $L_{k, n}$ влекут соответствующие результаты о распределении другой величины.

После значительного перерыва (в 1942 году локальный вариант равенства (1) был получен в [4]) форма графов Ферре изучалась Салаи и Тураном в серии статей [17-19]. В частности, они указали интервал (теорема II в [16]), с границами, зависящими от $n$, в котором $Z_{k, n}$ лежит с вероятностью, не меньшей $1-C n^{-7 / 4}$, где $C$ - некоторая положительная постоянная, а $k$ именяется в некоторой указанной области. Позднее в [7] было предпринято систематическое изучение случайных разбиений. С помощью объединения аналитических и вероятностных соображений были получены результаты о слабой сходимости распределений. Ради полноты мы приведем формулировки трех результатов статьи [7], касающихся формы случайного графа Ферре. Первый из этих результатов обобщает предельную теорему Эрдёша и Лехнера (1).

Теорема 1 ([7]). Для любого фиксированного положительного целого числа $k u$ любого фиксированного $v,-\infty<v<\infty$,

$$
\lim _{n \rightarrow \infty} \mathbf{P}\left\{\frac{\pi L_{k, n}}{(6 n)^{1 / 2}}-\log \frac{(6 n)^{1 / 2}}{\pi}<v\right\}=\int_{-\infty}^{v} \frac{\exp \left(-e^{-w}-k w\right)}{(k-1) !} d w
$$

Второй результат описывает поведение умеренно больших частей случайного разбиения, для которых имеет место сходимость к гауссовскому распределению.

Теорема 2 ([7]). Пусть $s=s_{n} \rightarrow \infty u n \rightarrow \infty$ maк, чmo $s / n^{1 / 4} \rightarrow 0$. Тогда для любого фиксированного $v,-\infty<v<\infty$,

$$
\lim _{n \rightarrow \infty} \mathbf{P}\left\{\frac{\pi s^{1 / 2} L_{s, n}}{(6 n)^{1 / 2}}-s^{1 / 2} \log \frac{(6 n)^{1 / 2}}{\pi s}<v\right\}=\frac{1}{\sqrt{2 \pi}} \int_{-\infty}^{v} e^{-w^{2} / 2} d w
$$

Третий результат статьи [7] описывает величину столбцов наименьшей высоты в случайном графе Ферре.

Теорема 3 ([7]). Пусть $s=s_{n}$ таково, что при $n \rightarrow \infty$

$$
s_{n} / n^{1 / 2} \rightarrow \infty
$$


$u$

$$
\pi s_{n} /(6 n)^{1 / 2}-\frac{1}{2} \log n \rightarrow-\infty .
$$

Тогда для любого фиксированного $v,-\infty<v<\infty$,

$$
\begin{array}{r}
\lim _{n \rightarrow \infty} \mathbf{P}\left\{\left(L_{s, n}+\pi^{-1}(6 n)^{1 / 2} \log \left(1-\exp \left(-\pi s /(6 n)^{1 / 2}\right)\right)\right) /\left(\pi^{-1 / 2}(6 n)^{1 / 4} e^{-\pi s / 2(6 n)^{1 / 2}}\right)<v\right\} \\
=\frac{1}{\sqrt{2 \pi}} \int_{-\infty}^{v} e^{-w^{2} / 2} d w
\end{array}
$$

Недавно Питтель [13] обобщил основную идею доказательства теоремы 3. Он подтвердил гипотезу, выдвинутую Арратия и Таваре в [3] в терминах рассотяния по вариации. Его результат показывает, что второе условие теоремы 3 можно заменить более общим условием, расширяющим область допустимых значений $s$. Питтель указал также вероятностные границы для $L_{s, n}$ во всей области изменения $s$ и получил результаты, аналогичные результатам Салаи и Турана [16-18]. Таким образом, статья Питтеля [13] показывает, что следовало бы заполнить пробел в исследованиях графов Ферре, изучив поведение частей порядка $O\left(n^{1 / 2}\right)$ случайного разбиения, и тем самым закончить изучение поведения случайных графов Ферре в терминах слабой сходимости распределений их характеристик к нетривиальным предельным распределениям. Цель настоящей статьи доказать следующую предельную теорему, которая вместе с теоремами 1 и 2 из [7] завершает описание асимптотического поведения случайного графа Ферре.

Теорема 4. Пусть

$$
\begin{aligned}
\lambda & =\pi s /(6 n)^{1 / 2} \\
\mu_{n}(\lambda) & =-\frac{(6 n)^{1 / 2}}{\pi} \log \left(1-e^{-\lambda}\right), \\
C(\lambda) & =-\frac{\sqrt{3} \log \left(1-e^{-\lambda}\right)}{\pi e^{-\lambda / 2}\left(1-e^{-\lambda}\right)^{1 / 2}}, \\
\sigma_{n}^{2}(\lambda) & =\frac{(6 n)^{1 / 2} e^{-\lambda}\left(1-C^{2}(\lambda)\right.}{\pi\left(1-e^{-\lambda}\right)} .
\end{aligned}
$$

Предположим, что целые $s=s_{n}$ таковы, что

$$
\lambda \leqslant \frac{1}{2} \log n-\log \log n-a_{n},
$$

где $a_{n} \rightarrow \infty$ как угодно медленно при $n \rightarrow \infty, u$

$$
\lambda>\lambda_{0}
$$

где $\lambda_{0}$ - единственный коренъ уравнения $C(\lambda)=1$.

Тогда для любого фиксированного $v,-\infty<v<\infty$,

$$
\lim _{n \rightarrow \infty} \mathbf{P}\left\{\left(L_{s, n}-\mu_{n}(\lambda)\right) / \sigma_{n}(\lambda)<v\right\}=\frac{1}{\sqrt{2 \pi}} \int_{-\infty}^{v} e^{-w^{2} / 2} d w
$$


Замечание 1. Если $\lambda \rightarrow \infty$, то $-\log \left(1-e^{-\lambda}\right) \sim e^{-\lambda}$ и $C(\lambda) \sim \sqrt{3} e^{-\lambda / 2} / \pi \rightarrow 0$. Кроме того, из первого условия теоремы 4 следует второе условие теоремы 3 . Таким образом, теорема 3 есть часть теоремы 4 . Нетрудно также проверить, что $C(\lambda) \rightarrow \infty$ при $\lambda \rightarrow 0$ и $C(\lambda)$ строго убывает при возрастании $\lambda$. Поэтому уравнение $C(\lambda)=1$ имеет единственный корень.

Замечание 2. Хванг недавно доказал [10] локальный вариант теоремы 2 в несколько более узкой области изменения $s$.

Замечание 3. Прямым следствием свойства двойственности графов Ферре является возможность переформулировать теорему 4 в терминах случайных величин $Z_{s, n}$. Ниже мы будем существенно пользоваться этим фактом при выводе основного результата с использованием тождества для производящей функции для $Z_{s, n}$ (см. лемму 1).

Замечание 4. Теоремы 1-3 были доказаны вероятностным методом. Чтобы описать основную идею доказательства, рассмотрим число $X_{j, n}$ частей, равных $j$, в случайном разбиении числа $n$. Оказывается, что совместное распределение $X_{j, n}$, $j=1, \ldots, n$, совпадает с совместным распределением первых $n$ членов последовательности $\left\{Z_{j}\right\}$, где $Z_{j}$ - независимые геометрически распределенные с параметрами $e^{-\pi j /(6 n)^{1 / 2}}$ случайные величины, при условии, что

$$
Z_{1}+2 Z_{2}+\ldots+n Z_{n}=n .
$$

Этот факт позвляет сводить задачи об асимптотическом поведении случайных разбиений к эквивалентным задачам для функций от случайных величин $Z_{j}, j=$ $1, \ldots, n$. Указанная выше связь играет важную роль в доказательствах основных резульаттов статей [7] и [13]. Подобный подход был впервые предложен Колчиным [11], применявшим его при изучении других комбинаторных объектов (подстановок, отображений, деревьев, лесов). В отличие от приведенных выше исследований в настоящей статье используется аналитический метод, основанный на применении метода перевала в форме, предлдоженной Нейманом [9]. Мы показываем, что основные производящие функции рассматриваемых в статье величин обладают свойствами, близкими свойствам рядов допустимых по Нейману функций (см., например, леммы 2, 3 и доказательство малости $J_{2}(y)$ ). Поскольку мы используем производящие функции моментов, мы также в доказательствах опираемся на теорему Куртисса, аналог теоремы непрерывности Леви [5].

Статья построена следующим образом. Раздел 2 содержит вспомогательные результаты об аналитических и асимптотических свойствах производящих функций и о расходящихся рядах, которые используются в дальнейшем. Доказательство теоремы 4 разбито на две части и изложено в разделах 3 и 4 . В разлеле 3 найден основной вклад в асимптотику изучаемой вероятности, а в разделе 4 оценивается остаточный член.

\section{2. Производящие функции и вспомогательные результаты}

Начнем с производящей функции $g(x)$ последовательности $\{p(n)\}$, где $p(n)$ обозначает общее число разбиений положительного целого числа $n$. Хорошо известно, что 
при $|x|<1$ производящая функция $g(x)$ может быть записана в виде

$$
g(x)=1+\sum_{n=1}^{\infty} p(n) x^{n}=\prod_{m \geqslant 1}\left(1-x^{m}\right)^{-1}
$$

(см., например, гл. 1 в [2]).

Пусть $a(n, k, s)$ обозначает число разбиений $n$, имеющих ровно $k$ частей, больших $s-1$. Рассуждая так же, как при доказательстве представления (5), можно показать, что

$$
\begin{aligned}
1+\sum_{n=1}^{\infty} \sum_{k=0}^{n} a(n, k, s) x^{n} y^{k} & =\prod_{m=1}^{s-1}\left(1-x^{m}\right)^{-1} \prod_{m=s}^{\infty}\left(1-x^{m} y\right)^{-1} \\
& =g(x) \prod_{m \geqslant s} \frac{1-x^{m}}{1-x^{m} y}
\end{aligned}
$$

(см. также [17], стр. 382-383). Из определения $Z_{s, n}$, приведенного во введении, следует, что

$$
\frac{1}{p(n)} \sum_{k=0}^{n} a(n, k, s) y^{k}=\mathbf{E} y^{Z_{s, n}} .
$$

Это равенство приводит к следующему основному тождеству для производящей функции, которое ниже будет использовано как отправная точка асимптотического анализа.

Лемма 1. При $|x|<1 u|y| \leqslant 1$

$$
1+\sum_{n=1}^{\infty} x^{n} p(n) \mathrm{E} y^{Z_{s, n}}=g(x) \prod_{m \geqslant s} \frac{1-x^{m}}{1-x^{m} y}
$$

где $g(x)$ определена в (5).

Приступим к асимптотической оценке $g(x)$. Введем дополнительные обозначения. Пусть $\lambda=\lambda_{n}$ - последовательность, удовлетворяющая обоим условиям теоремы 4 , и определим последовательность $\left\{r_{n}\right\}$ так, что

$$
r_{n}=1-\pi /(6 n)^{1 / 2}-\lambda / 2 n+O\left(\lambda^{2} / n^{3 / 2}\right)
$$

при $n \rightarrow \infty$. Для краткости введем также обозначение

$$
b\left(r_{n}\right)=\frac{\pi^{2}}{3\left(1-r_{n}\right)^{3}}
$$

Для последующего асимптотического анализа важно изучить поведение производящей функции $g(x)$ в окрестности $x=1$. Следующая лемма содержит результат, который впоследствии, в доказательстве теоремы 4, используется для получения главного члена асимптотики изучаемой вероятности.

Лемма 2. Если $r_{n}$ удовлетворяет соотношению (6), то при $n \rightarrow \infty$ равномерно no $|\theta| \leqslant \delta_{n}$

$$
g\left(r_{n} e^{i \theta}\right) e^{-i \theta n}=g\left(r_{n}\right) e^{-\theta^{2} b\left(r_{n}\right) / 2}\left(1+O\left(1 / \log ^{3} n\right)\right),
$$


где $b\left(r_{n}\right)$ определено в (7) $u$

$$
\delta_{n}=n^{-2 / 3} / \log n
$$

Доказателъство. Отправной точкой доказательства будет принадлежащее Фрейману простое представление $g\left(e^{-z}\right)$ при $z \rightarrow 0$ (см. раздел 2.7 в [14])

$$
g\left(e^{-z}\right)=\exp \left\{\frac{\pi^{2}}{6 z}+\frac{1}{2} \log \frac{z}{2 \pi}+O(|z|)\right\}
$$

равномерное в области

$$
\{z: \Im(z) \leqslant \varepsilon \Re(z), \Re(z)>0\}
$$

где $\varepsilon>0$ фиксировано. Если в этой формуле положить $x=e^{-z}$, то $x \rightarrow 1$ и

$$
z=-\log (1-(1-x))=1-x+\frac{1}{2}(1-x)^{2}+O\left(|1-x|^{3}\right)
$$

Поэтому в терминах $x$ соотношение (9) принимает следующий вид: при $x \rightarrow 1$

$$
g(x)=\exp \left\{\frac{\pi^{2}}{6(1-x)}-\frac{\pi^{2}}{12}+\frac{1}{2} \log \frac{1-x}{2 \pi}+O(|1-x|)\right\}
$$

При $x=r_{n} e^{i \theta}$

$$
\Re(z)=-\log r_{n}, \quad \Im(z)=-\theta .
$$

Таким образом, (10) справедливо, если $|\theta| \leqslant \delta_{n}$, так как в силу (8) и (6)

$$
\delta_{n}<-\varepsilon \log r_{n} \sim \frac{\epsilon \pi}{(6 n)^{1 / 2}}
$$

для любого фиксированного $\varepsilon>0$. Легко проверить, что в силу (6) и (8)

$$
\left|1-r_{n} e^{i \theta}\right|=\frac{\pi}{(6 n)^{1 / 2}}\left(1+o\left(n^{-1 / 3}\right)\right)
$$

так что остаточный член в $(10)$ есть $O\left(n^{-1 / 2}\right)$. Полагая вначале $x=r_{n} e^{i \theta}$, а затем $x=r_{n}$ в $(10)$, деля первое выражение на второе и разлагая $\left(1-r_{n} e^{i \theta}\right)^{-1}$ в ряд 
Тейлора, находим, что

$$
\begin{aligned}
\frac{e^{-i \theta n} g\left(r_{n} e^{i \theta}\right)}{g\left(r_{n}\right)}=\left(\frac{1-r_{n} e^{i \theta}}{1-r_{n}}\right)^{1 / 2} & \times \exp \left\{\frac{\pi^{2}}{6}\left(\frac{1}{1-r_{n} e^{i \theta}}-\frac{1}{1-r_{n}}\right)-i \theta n+O\left(n^{-1 / 2}\right)\right\} \\
= & \left.\frac{1-r_{n}+O(|\theta|)}{1-r_{n}}\right)^{1 / 2} \\
& \times \exp \left\{\frac { \pi ^ { 2 } } { 6 } \left(\frac{r_{n}\left(e^{i \theta}-1\right)}{\left(1-r_{n}\right)^{2}}+\frac{r_{n}^{2}\left(e^{i \theta}-1\right)^{2}}{\left(1-r_{n}\right)^{3}}\right.\right. \\
& \left.\left.+O\left(\frac{\left|e^{i \theta}-1\right|^{3}}{\left(1-r_{n}\right)^{4}}\right)\right)-i \theta n+O\left(n^{-1 / 2}\right)\right\} \\
= & \exp \left\{i \theta\left(\frac{\pi^{2} r_{n}}{6\left(1-r_{n}\right)^{2}}-n\right)-\frac{\theta^{2} \pi^{2} r_{n}}{12\left(1-r_{n}\right)^{2}}\left(1+\frac{2 r_{n}}{1-r_{n}}\right)\right. \\
& \left.+O\left(\frac{|\theta|^{4}}{\left(1-r_{n}\right)^{3}}\right)+O\left(\frac{|\theta|^{3}}{\left(1-r_{n}\right)^{4}}\right)+O\left(n^{-1 / 2}\right)\right\} \\
& \times\left(1+O\left(\delta_{n}\left(1-r_{n}\right)^{-1}\right)\right)^{1 / 2} \\
= & \exp \left\{i \theta\left(\frac{\pi^{2} r_{n}}{6\left(1-r_{n}\right)^{2}}-n\right)-\frac{\theta^{2} \pi^{2} r_{n}}{12\left(1-r_{n}\right)^{2}}\left(1+\frac{2 r_{n}}{1-r_{n}}\right)\right. \\
& \left.+O\left(\frac{\delta_{n}^{4}}{\left(1-r_{n}\right)^{3}}\right)+O\left(\frac{\delta_{n}^{3}}{\left(1-r_{n}\right)^{4}}\right)+O\left(n^{-1 / 2}\right)\right\} \\
& \times\left(1+O\left(\delta_{n}\left(1-r_{n}\right)^{-1}\right)\right) .
\end{aligned}
$$

Заменим теперь $r_{n}$ в (11) на асимптотическое предствление $(6), \delta_{n}$ на (8) и $\lambda$ на оценку сверху из первого условия теоремы 4 . В результате множитель вне экспоненты окажется равным

$$
1+O\left(\delta_{n}\left(1-r_{n}\right)^{-1}\right)=1+O\left(\delta_{n} n^{1 / 2}\right)=1+O\left(n^{-1 / 6} / \log n\right) .
$$

Член, содержащий $i \theta$, пренебрежимо мал, так как

$$
\begin{aligned}
i \theta\left(\frac{\pi^{2}}{6\left(1-r_{n}\right)^{2}}-n\right) & =i \theta\left(\frac{\pi^{2}}{6\left(\pi^{2} /(6 n)+O\left(\lambda / n^{3 / 2}\right)\right)}-n\right) \\
& =i \theta\left(\frac{n}{1+O\left(\lambda / n^{1 / 2}\right)}-n\right)=i \theta\left(n\left(1+O\left(\lambda / n^{1 / 2}\right)\right)-n\right) \\
& =O\left(\delta_{n} \lambda n^{1 / 2}\right)=O\left(n^{-1 / 6}\right) .
\end{aligned}
$$

Для того чтобы найти коэффициент при $-\theta^{2}$ в последней экспоненте в (11), заметим прежде всего, что из (6) и (7) следует, что при $n \rightarrow \infty$

$$
b\left(r_{n}\right)=(2 / \pi) 6^{1 / 2} n^{3 / 2}\left(1+O\left(\lambda / n^{1 / 2}\right)\right) .
$$

Поэтому

$\frac{\pi^{2} r_{n}}{12\left(1-r_{n}\right)^{2}}\left(1+\frac{2 r_{n}}{1-r_{n}}\right)=\frac{\pi^{2} r_{n}^{2}}{6\left(1-r_{n}\right)^{3}}+O(n)=\frac{\pi^{2}}{6\left(1-r_{n}\right)^{3}}+O(n)=\frac{1}{2} b\left(r_{n}\right)+O(n)$. 
Поскольку $O\left(n|\theta|^{2}\right)=O\left(n \delta_{n}^{2}\right)=O\left(n^{-1 / 3} / \log ^{2} n\right)$, получаем, что

$$
-\frac{\theta^{2} \pi^{2}}{12\left(1-r_{n}\right)^{2}}\left(1+\frac{2 r_{n}}{1-r_{n}}\right)=-\frac{1}{2} \theta^{2} b\left(r_{n}\right)+O\left(n^{-1 / 3} / \log ^{2} n\right) .
$$

Другие остаточные члены в экспоненте оцениваются аналогично:

$$
\begin{aligned}
& O\left(\delta_{n}^{4}\left(1-r_{n}\right)^{-3}\right)=O\left(n^{-7 / 6} / \log ^{4} n\right), \\
& O\left(\delta_{n}^{3}\left(1-r_{n}\right)^{-4}\right)=O\left(1 / \log ^{3} n\right) .
\end{aligned}
$$

Подставляя оценки (12), (13), (15)-(17) в (11), получаем требуемое соотношение.

При изучении поведения $g\left(r_{n} e^{i \theta}\right)$ вне области $-\delta_{n}<\theta<\delta_{n}$ нам потребуется следующая лемма.

Лемма 3. Если $r_{n}$ удовлетворяет (6) $u \delta_{n} \leqslant \Delta_{n}=o\left(n^{-1 / 2}\right)$, то

$$
\frac{1}{\left|1-r_{n} e^{i \theta}\right|}-\frac{1}{1-r_{n}} \leqslant-\frac{3 \sqrt{6} n^{3 / 2} \Delta_{n}^{2}}{\pi^{3}}+O\left(\lambda^{2}\right)
$$

равномерно относителъно $|\theta| \geqslant \Delta_{n}$.

Доказательство. С использованием (6) и (8) находим, что

$$
\begin{aligned}
\left|1-r_{n} e^{i \theta}\right|= & \left(1+r_{n}^{2}-2 r_{n} \cos \theta\right)^{1 / 2} \geqslant\left(1+r_{n}^{2}-2 r_{n} \cos \Delta_{n}\right)^{1 / 2} \\
= & \left(2-2 \pi /(6 n)^{1 / 2}+\pi^{2} /(6 n)-\lambda / n+O\left(\lambda^{2} / n^{3 / 2}\right)\right. \\
& \left.\quad-2\left(1-\pi /(6 n)^{1 / 2}-\lambda /(2 n)+O\left(\lambda^{2} / n^{3 / 2}\right)\right)\left(1-\Delta_{n}^{2} / 2+O\left(\Delta_{n}^{4}\right)\right)\right)^{1 / 2} \\
= & \left(\Delta_{n}^{2}+\pi^{2} /(6 n)+O\left(\lambda^{2} / n^{3 / 2}\right)\right)^{1 / 2} .
\end{aligned}
$$

Отсюда следует, что

$$
\begin{aligned}
\frac{1}{\mid 1-r_{n} e^{i \theta \mid}-\frac{1}{1-r_{n}} \leqslant} \leqslant & \frac{1}{\left(\Delta_{n}^{2}+\pi^{2} /(6 n)+O\left(\lambda^{2} / n^{3 / 2}\right)\right)^{1 / 2}} \\
& -\frac{1}{\pi /(6 n)^{1 / 2}+\lambda /(2 n)+O\left(\lambda^{2} / n^{3 / 2}\right)} \\
= & \frac{(6 n)^{1 / 2}}{\pi}\left(\frac{1}{\left(1+6 n \Delta_{n}^{2} / \pi^{2}+O\left(\lambda / n^{1 / 2}\right)\right)^{1 / 2}}\right. \\
& \left.-\frac{1}{1+(\lambda / 2 \pi)(6 / n)^{1 / 2}+O\left(\lambda^{2} / n\right)}\right) \\
= & \frac{(6 n)^{1 / 2}}{\pi}\left(-\frac{1}{2}\left(\frac{6 n \Delta_{n}^{2}}{\pi^{2}}+O\left(\frac{\lambda^{2}}{n^{1 / 2}}\right)\right)+\frac{\lambda}{2 \pi}\left(\frac{6}{n}\right)^{1 / 2}\right. \\
& \left.+O\left(n^{2} \Delta_{n}^{4}\right)+O\left(\lambda^{2} \Delta_{n}^{2} n^{1 / 2}\right)+O\left(\lambda^{2} / n\right)\right) \\
= & \frac{(6 n)^{1 / 2}}{\pi}\left(-3 n \Delta_{n}^{2} / \pi^{2}+O\left(\lambda^{2} / n^{1 / 2}\right)+(\lambda / 2 \pi)(6 / n)^{1 / 2}\right) \\
= & -\frac{(6 n)^{1 / 2} 3 n \Delta_{n}^{2}}{\pi^{3}}+\frac{3 \lambda}{\pi^{2}}+O\left(\lambda^{2}\right)=-\frac{3 \sqrt{6} n^{3 / 2} \Delta_{n}^{2}}{\pi^{3}}+O\left(\lambda^{2}\right) .
\end{aligned}
$$

Лемма доказана. 
Далее изучается асимптотическое поведение сумм

$$
d_{j}(x)=\sum_{m \geqslant s} \frac{x^{m j}}{\left(1-x^{m}\right)^{j}}, \quad j=1,2,3,
$$

и их производных $d_{1}^{\prime}(x), d_{1}^{\prime \prime}(x), d_{2}^{\prime}(x)$ для значений $s$ таких, как в теореме 4 , при $x=r_{n}$. Начнем с изучения асимптотики $d_{1}\left(r_{n}\right)$. Остальные функции могут быть проанализированы аналогичным образом, поэтому мы опускаем детали доказательств.

Логарифмируя обе части (6), получаем, что

$$
\log r_{n}=-\frac{\pi}{(6 n)^{1 / 2}}-\frac{1}{n}\left(\frac{\lambda}{2}+\frac{\pi^{2}}{12}\right)+O\left(\frac{\lambda^{2}}{n^{3 / 2}}\right) .
$$

Поэтому

$$
\begin{aligned}
& d_{1}\left(r_{n}\right)=\sum_{m \geqslant s} \frac{e^{m \log r_{n}}}{1-e^{m \log r_{n}}}=\frac{(6 n)^{1 / 2}}{\pi} \\
& \times \sum_{\pi m /(6 n)^{1 / 2} \geqslant \lambda} \frac{\pi \exp \left(-m \pi /(6 n)^{1 / 2}-m\left(\lambda / 2+\pi^{2} / 12\right) / n+O\left(\lambda^{2} m / n^{3 / 2}\right)\right)}{(6 n)^{1 / 2}\left(1-\exp \left(-m \pi /(6 n)^{1 / 2}-m\left(\lambda / 2+\pi^{2} / 12\right) / n+O\left(\lambda^{2} m / n^{3 / 2}\right)\right)\right)} .
\end{aligned}
$$

Рассматривая сумму в (19) как интегральную сумму, заменим ее соответствующим игнтегралом. Поэтому в силу мажорируемой сходимости (см., например, [15])

$$
\begin{aligned}
d_{1}\left(r_{n}\right)= & \frac{(6 n)^{1 / 2}}{\pi}\left(1+O\left(n^{-1 / 2}\right)\right) \\
& \times \int_{\lambda}^{\infty} \frac{e^{-u} \exp \left\{-(6 / n)^{1 / 2} u\left(\lambda / 2+\pi^{2} / 12\right) / \pi+O\left(\lambda^{2} u / n\right)\right\}}{1-e^{-u} \exp \left\{-(6 / n)^{1 / 2} u\left(\lambda / 2+\pi^{2} / 12\right) / \pi+O\left(\lambda^{2} u / n\right)\right\}} d u \\
= & \frac{(6 n)^{1 / 2}}{\pi} \int_{\lambda}^{\infty} \frac{e^{-u}}{1-e^{-u}} d u\left(1+O\left(\lambda / n^{1 / 2}\right)\right) \\
= & -\frac{(6 n)^{1 / 2}}{\pi} \log \left(1-e^{-\lambda}\right)\left(1+O\left(\lambda / n^{1 / 2}\right)\right) .
\end{aligned}
$$

Поэтому, заключаем, что

$$
d_{1}\left(r_{n}\right)=-\frac{(6 n)^{1 / 2}}{\pi} \log \left(1-e^{-\lambda}\right)\left(1+O\left(\lambda / n^{1 / 2}\right)\right)
$$

Рассуждая аналогичным образом, получаем, что

$$
\begin{aligned}
& d_{1}^{\prime}\left(r_{n}\right)=\sum_{m \geqslant s} \frac{m r_{n}^{m-1}}{\left(1-r_{n}^{m}\right)^{2}}=-\frac{6 n\left(1+O\left(\lambda / n^{1 / 2}\right)\right)}{\pi^{2}\left(1-e^{-\lambda}\right)} \log \left(1-e^{-\lambda}\right), \\
& d_{1}^{\prime \prime}\left(r_{n}\right)=\sum_{m \geqslant s} \frac{m^{2} r_{n}^{m-2}\left(1+r_{n}^{m}+\left(r_{n}^{m}-1\right) / m\right)}{\left(1-r_{n}^{m}\right)^{3}}=O\left(n e^{-\lambda}\right), \\
& d_{2}\left(r_{n}\right)=\frac{(6 n)^{1 / 2}}{\pi}\left(e^{-\lambda} /\left(1-e^{-\lambda}\right)+\log \left(1-e^{-\lambda}\right)+O\left(\lambda / n^{1 / 2}\right)\right), \\
& d_{2}^{\prime}\left(r_{n}\right)=\frac{2}{r_{n}} \sum_{m \geqslant s} \frac{m r_{n}^{2 m}}{\left(1-r_{n}^{m}\right)^{3}}=O\left(n e^{-\lambda}\right), \\
& d_{3}\left(r_{n}\right)=O\left(n^{1 / 2} e^{-\lambda}\right),
\end{aligned}
$$


Далее нам понадобится асимптотика коэффициентов $p(n)$ в представлении (5). Хотя формула Харди-Рамануджана [2] дает возможность достаточно точно вычислять $p(n)$, мы будем использовать этот результат в несколько иной форме. Одлыжко и Ричмонд доказали [13], что произв дящая функция $g(x)$, определенная в (5), принадлежит классу Неймана допустимых функций [9]. Поэтому общий результат Неймана верен для асимптотики чисел $p(n)$ разбиений $n$. Следующая лемма объединяет эти два результата.

Лемма 4 ([9, 13]). При $n \rightarrow \infty$

$$
p(n) \sim g\left(r_{n}\right) r_{n}^{-n} /\left(2 \pi \beta\left(r_{n}\right)\right)^{1 / 2},
$$

2de

$$
\begin{aligned}
\beta(r) & =r \frac{g^{\prime}(r)}{g(r)}+r^{2} \frac{g^{\prime \prime}(r)}{g(r)}-r^{2}\left(\frac{g^{\prime}(r)}{g(r)}\right)^{2} \\
& =\sum_{m=1}^{\infty} \frac{m r^{m}}{\left(1-r^{m}\right)^{2}}+2 \sum_{m=1}^{\infty} \frac{m r^{m+1}}{\left(1-r^{m}\right)^{3}}
\end{aligned}
$$

$u r_{n}$ удовлетворяет уравнению

$$
r g^{\prime}(r) / g(r)=n
$$

при достаточно больших $n$.

Замечание 5. Поскольку при $r \rightarrow 1-$

$$
\frac{r g^{\prime}(r)}{g(r)}=\sum_{m=1}^{\infty} \frac{r^{m}}{\left(1-r^{m}\right)^{2}}=\frac{\pi^{2}}{6(1-r)^{2}}(1+o(1))
$$

нетрудно проверить, что $r_{n}$, определенное уравнением (27), удовлетворяет соотношению (6). Кроме того, используя (26), можно показать, что при $r \rightarrow 1-$

$$
\beta(r)=\frac{\pi^{2}}{3(1-r)^{3}}\left(1+O\left((1-r)^{1 / 2}\right)\right),
$$

так что в силу (7)

$$
b\left(r_{n}\right) / \beta\left(r_{n}\right)=1+O\left(\left(1-r_{n}\right)^{1 / 2}\right)
$$

при $n \rightarrow \infty$.

Начнем с того, что для определения коэффициентов в тождестве леммы 1 , мы применим формулу Коши с контуром интегрирования $\left\{x: x=r_{n} e^{i \theta},-\pi<\theta \leqslant \pi\right\}$. Таким образом,

$$
r_{n}^{n} p(n) \mathbf{E} y^{Z_{s, n}}=\frac{1}{2 \pi} \int_{-\pi}^{\pi} \exp \left(G\left(r_{n} e^{i \theta}, y\right)-i \theta n\right) d \theta
$$

где

$$
G(x, y)=\log g(x)+\sum_{m \geqslant s} \log \frac{1-x^{m}}{1-x^{m} y}
$$


причем берется основная ветвь логарифмической функции так, что $\log g(x)>0$, если $0<x<1$. Разобьем (29) на две части, полагая

$$
r_{n}^{n} p(n) \mathbf{E} y^{Z_{s, n}}=J_{1}(y)+J_{2}(y)
$$

где

$$
\begin{aligned}
& J_{1}(y)=\frac{1}{2 \pi} \int_{-\delta_{n}}^{\delta_{n}} \exp \left(G\left(r_{n} e^{i \theta}, y\right)-i \theta n\right) d \theta \\
& J_{2}(y)=\frac{1}{2 \pi} \int_{\delta_{n}<|\theta| \leqslant \pi} \exp \left(G\left(r_{n} e^{i \theta}, y\right)-i \theta n\right) d \theta
\end{aligned}
$$

и $\delta_{n}$ определяется равентством (8). Асимптотический анализ $J_{j}(y), j=1,2$, проводится в двух следующих разделах.

\section{3. Асимптотическая оценка $J_{1}(y)$}

Заметим, что

$$
G(x, 1)=\log g(x)
$$

и

$$
\left.\frac{\partial^{j}}{\partial y^{j}} G(x, y)\right|_{y=1}=d_{j}(x), \quad j=1,2,3
$$

(см. (30)). Используя разложение в ряд Тейлора, находим, что

$$
\begin{aligned}
G\left(r_{n} e^{i \theta}, y\right)=\log g\left(r_{n} e^{i \theta}\right)+(y-1) d_{1}\left(r_{n} e^{i \theta}\right) & +\frac{1}{2}(y-1)^{2} d_{2}\left(r_{n} e^{i \theta}\right) \\
& +O\left(|y-1|^{3} \sum_{m \geqslant s} \frac{r_{n}^{3 m}}{\left|1-r_{n}^{m} e^{i \theta m} y_{0}\right|}\right)
\end{aligned}
$$

где $0<y_{0}<y$. Ясно, что остаточный член в этой формуле есть $O\left(|y-1|^{3} d_{3}\left(r_{n}\right)\right)$. Поэтому, применяя лемму 2 , находим, что

$$
\begin{aligned}
J_{1}(y)=(1 & \left.+O\left(1 / \log ^{3} n\right)\right) \frac{g\left(r_{n}\right)}{2 \pi} \int_{-\delta_{n}}^{\delta_{n}} e^{-\theta^{2} b\left(r_{n}\right) / 2} \exp \left((y-1) d_{1}\left(r_{n} e^{i \theta}\right)\right. \\
& \left.+(y-1)^{2} d_{2}\left(r_{n} e^{i \theta}\right) / 2+O\left(|y-1|^{3} d_{3}\left(r_{n}\right)\right)\right) d \theta \\
=(1 & \left.+O\left(1 / \log ^{3} n\right)\right) \frac{g\left(r_{n}\right)}{2 \pi b^{1 / 2}\left(r_{n}\right)} \int_{-\delta_{n} b^{1 / 2}\left(r_{n}\right)}^{\delta_{n} b^{1 / 2}\left(r_{n}\right)} e^{-t^{2} / 2} \exp \left((y-1) d_{1}\left(r_{n} e^{i t / b^{1 / 2}\left(r_{n}\right)}\right)\right. \\
& \left.+(y-1)^{2} d_{2}\left(r_{n} e^{i t / b^{1 / 2}\left(r_{n}\right)}\right) / 2+O\left(|y-1|^{3} d_{3}\left(r_{n}\right)\right)\right) d t .
\end{aligned}
$$

Заметим, что для получения последней оценки используется замена переменной интегрирования $\theta=t / b^{1 / 2}\left(r_{n}\right)$. Снова применяя разложение в ряды Тейлора функций 
$d_{1}$ и $d_{2}$ и используя $(8),(21),(22)$ и $(24)$, получаем, что

$$
\begin{aligned}
d_{1}\left(r_{n} e^{i t / b^{1 / 2}\left(r_{n}\right)}\right) & =d_{1}\left(r_{n}\right)+r_{n} d_{1}^{\prime}\left(r_{n}\right)\left(e^{i t / b^{1 / 2}\left(r_{n}\right)}-1\right)+O\left(d_{1}^{\prime \prime}\left(r_{n}\right) \delta_{n}^{2}\right) \\
& =d_{1}\left(r_{n}\right)+r_{n} d_{1}^{\prime}\left(r_{n}\right) i t / b^{1 / 2}\left(r_{n}\right)+O\left(\left(d_{1}^{\prime}\left(r_{n}\right)+d_{1}^{\prime \prime}\left(r_{n}\right)\right) \delta_{n}^{2}\right) \\
& =d_{1}\left(r_{n}\right)+r_{n} d_{1}^{\prime}\left(r_{n}\right) i t / b^{1 / 2}\left(r_{n}\right)+O\left(n^{-1 / 12} \log ^{3} n\right), \\
d_{2}\left(r_{n} e^{i t / b^{1 / 2}\left(r_{n}\right)}\right) & =d_{2}\left(r_{n}\right)+O\left(d_{2}^{\prime}\left(r_{n}\right) \delta_{n}\right) \\
& =d_{2}\left(r_{n}\right)+O\left(n^{1 / 3} e^{-\lambda} / \log n\right),
\end{aligned}
$$

В (34) положим $y=e^{w / \sigma_{n}^{\prime}(\lambda)}$, где

$$
{\sigma_{n}^{\prime}}^{2}(\lambda)=\sigma_{n}^{2}(\lambda) /(1-C(\lambda))
$$

а $C(\lambda)$ и $\sigma_{n}(\lambda)$ определены формулами (3) и (4), и оценим $J_{1}(y)$ при произвольном фиксированном $w,-\infty<w<\infty$.

Очевидно, что при $n \rightarrow \infty$

$$
\begin{aligned}
y-1 & =w / \sigma_{n}^{\prime}(\lambda)+w^{2} /\left(2{\sigma_{n}^{\prime}}^{2}(\lambda)\right)+O\left(\left(\sigma_{n}^{\prime}\right)^{-3}(\lambda)\right) \\
(y-1)^{2} & =w^{2} /{\sigma_{n}^{\prime}}^{2}(\lambda)+O\left(\left(\sigma_{n}^{\prime}\right)^{-3}(\lambda)\right) \\
(y-1)^{3} & =O\left(\left(\sigma_{n}^{\prime}\right)^{-3}(\lambda)\right)
\end{aligned}
$$

Из (3) и (4) следуют также оценки

$$
\begin{array}{cl}
{\sigma_{n}^{\prime}}^{2}(\lambda) \sim(6 n)^{1 / 2} e^{-\lambda} / \pi, & \lambda \rightarrow \infty, \\
C_{1} n^{1 / 2} \leqslant{\sigma_{n}^{\prime}}^{2}(\lambda) \leqslant C_{2} n^{1 / 2}, & \lambda \geqslant \lambda_{0}>0, \\
0<\lambda \leqslant \lambda=O(1), &
\end{array}
$$

где $C_{1}$ и $C_{2}$ - некоторые постоянные и неравенства выполняются, начиная с некоторого $n$, равномерно по $\lambda$. Используя (8) и (14), находим асимптотику границ интегрирования в интеграле в (34), а именно, при $n \rightarrow \infty$

$$
\delta_{n} b^{1 / 2}\left(r_{n}\right) \sim c n^{1 / 12} \log n
$$

где $c=(2 / \pi)^{1 / 2} 6^{1 / 4}$.

Поэтому, подставляя (35)-(43) в (34), после некоторых алгебраических преобразований, находим, что

$$
J_{1}\left(e^{w / \sigma_{n}^{\prime}(\lambda)}\right)=\left(1+O\left(1 / \log ^{3} n\right)\right) \frac{g\left(r_{n}\right)}{2 \pi b^{1 / 2}\left(r_{n}\right)} \int_{-c n^{1 / 12} \log n}^{c n^{1 / 12} \log n} \exp \left(-t^{2} / 2+W_{n}(t, w)\right) d t
$$

где

$$
\begin{aligned}
W_{n}(t, w)=\left(w / \sigma_{n}^{\prime}(\lambda)\right)\left(d_{1}\left(r_{n}\right)-\mu_{n}(\lambda)\right)+i t w r_{n} d_{1}^{\prime}\left(r_{n}\right) /\left(\sigma_{n}^{\prime}(\lambda) b^{1 / 2}\left(r_{n}\right)\right) \\
+\left(w^{2} /\left(2{\sigma_{n}^{\prime}}^{2}(\lambda)\right)\right)\left(d_{1}\left(r_{n}\right)+d_{2}\left(r_{n}\right)\right)+U_{n}(w)
\end{aligned}
$$


и в силу $(3),(4),(8),(21),(36)$ и $(41)$ остаточный член $U_{n}(w)$ есть $O\left(n^{-1 / 6}\right)$ при фиксированном $w$. Кроме того, из (20) и (41) следует, что

$$
\left(w / \sigma_{n}^{\prime}(\lambda)\right)\left(d_{1}\left(r_{n}\right)-\mu_{n}(\lambda)\right)=O\left(\lambda n^{-1 / 4} \log \left(1-e^{-\lambda}\right)\right)=O\left(n^{-1 / 4} \log ^{2} n\right) .
$$

Таким образом, возвращаясь к (45), мы можем переписать это соотношение при фиксированоом $w$ и $n \rightarrow \infty$ в более удобной форме:

$$
W_{n}(t, w)=\frac{i t r_{n} d_{1}^{\prime}\left(r_{n}\right)}{\sigma_{n}^{\prime}(\lambda) b^{1 / 2}\left(r_{n}\right)}+\frac{w^{2}}{2{\sigma_{n}^{\prime}}^{2}(\lambda)}\left(d_{1}\left(r_{n}\right)+d_{2}\left(r_{n}\right)\right)+O\left(n^{-1 / 6}\right) .
$$

Теперь нужно найти оценки коэффициентов при $i t w$ и $w^{2} / 2$ в $W_{n}(t, w)$. Напомним, что величина $C(\lambda)$ определено в (3) и она вносит ненулевой вклад в (4), если $\lambda$ не стремится к бесконечности (см. замечание 1). Используя $(21),(3),(4),(14)$ и (6), легко находим, что

$$
i t w r_{n} d_{1}^{\prime}\left(r_{n}\right) /\left(\sigma_{n}^{\prime}(\lambda) b^{1 / 2}\left(r_{n}\right)\right)=\left(1+O\left(\lambda / n^{1 / 2}\right)\right) i t w C(\lambda) .
$$

Далее, из (20) и (23) следует, что

$$
d_{1}\left(r_{n}\right)+d_{2}\left(r_{n}\right)=\frac{(6 n)^{1 / 2} e^{-\lambda}}{\pi\left(1-e^{-\lambda}\right)}\left(1+O\left(\lambda / n^{1 / 2}\right)\right),
$$

поэтому так же, как и (47), получаем, что

$$
\frac{w^{2}}{2{\sigma_{n}^{\prime}}^{2}(\lambda)}\left(d_{1}\left(r_{n}\right)+d_{2}\left(r_{n}\right)\right)=\frac{w^{2}}{2}+O\left(\lambda / n^{1 / 2}\right) .
$$

Прежде чем приступить к получению окончательной оценки заметим, что в силу (31) удобно разделить $J_{1}\left(e^{w / \sigma_{n}^{\prime}(\lambda)}\right) e^{-w \mu_{n}(\lambda) / \sigma_{n}^{\prime}(\lambda)}$ на $r_{n}^{n} p(n)$. Подставляя вначале (46)-(48) в (44) и учитывая лемму 4 и $(28)$, получаем, что

$$
\begin{aligned}
& J_{1}\left(e^{w / \sigma_{n}^{\prime}(\lambda)}\right) \frac{e^{-\mu_{n}(\lambda) w / \sigma_{n}^{\prime}(\lambda)}}{p(n) r_{n}^{n}} \\
& =\left(1+O\left(1 / \log ^{3} n\right)\right) \frac{g\left(r_{n}\right) r_{n}^{-n}}{2 \pi p(n) b^{1 / 2}\left(r_{n}\right)} \\
& \quad \times \int_{-c n^{1 / 12} \log n}^{c n^{1 / 12} \log n} \exp \left\{-t^{2} / 2+i t w C(\lambda)+w^{2} / 2+O\left(n^{-1 / 6}\right)\right\} d t \\
& =\left(1+O\left(1 / \log ^{3} n\right)\right)(2 \pi)^{-1 / 2}\left(\beta\left(r_{n}\right) / b\left(r_{n}\right)\right)^{1 / 2} \\
& \quad \times \exp \left\{w^{2} / 2+O\left(n^{-1 / 6}\right)\right\} \int_{-c n^{1 / 12} \log n}^{c n^{1 / 12} \log n} \exp \left\{-t^{2} / 2+i t w C(\lambda)\right\} d t \\
& =\left(1+O\left(1 / \log ^{3} n\right)\right)(2 \pi)^{-1 / 2} \exp \left\{w^{2} / 2+O\left(n^{-1 / 6}\right)\right\} \\
& \quad \times \int_{-c n^{1 / 12} \log n}^{c n^{1 / 12} \log n} \exp \left\{-t^{2} / 2+i t w C(\lambda)\right\} d t \\
& =\left(1+O\left(1 / \log ^{3} n\right)\right)(2 \pi)^{-1 / 2} \exp \left\{w^{2} / 2+O\left(n^{-1 / 6}\right)\right\} \\
& \quad \times\left(\int_{-\infty}^{\infty} \exp ^{2}\left\{-t^{2} / 2+i t w C(\lambda)\right\} d \dot{t}+O\left(e^{\left.\left.-c n^{1 / 6} \log ^{2} n /\left(n^{1 / 12} \log n\right)\right)\right) .}\right.\right.
\end{aligned}
$$


В последней оценке мы использовали хорошо известную асимптотику при $z \rightarrow \infty$ интеграла

$$
2 \pi^{-1 / 2} \int_{z}^{\infty} e^{-t^{2}} d t
$$

(см., например, гл. 7 в [1]). Далее, ясно, что

$$
\frac{1}{\sqrt{2 \pi}} \int_{-\infty}^{\infty} \exp \left\{-t^{2} / 2+i t w C(\lambda)\right\} d t=e^{-w^{2} C^{2}(\lambda) / 2} .
$$

Записывая остаточный член в (49) как $O\left(1 / \log ^{3} n\right)$ и подставляя (50) в (49), окончательно получаем, что

$$
J_{1}\left(e^{w / \sigma_{n}^{\prime}(\lambda)}\right) \frac{e^{-\mu_{n}(\lambda) w / \sigma_{n}^{\prime}(\lambda)}}{p(n) r_{n}^{n}}=e^{w^{2}\left(1-C^{2}(\lambda) / 2\right.}+O\left(1 / \log ^{3} n\right) .
$$

Это завершает асимптотическую оценку интеграла $J_{1}(y)$.

\section{4. Асимптотическая оценка $J_{2}(y)$}

Начнем с простого тождества

$$
\begin{aligned}
\log g(x) & =\sum_{m=1}^{\infty} \log \frac{1}{1-x^{m}}=\sum_{m=1}^{\infty} \sum_{l=1}^{\infty} \frac{x^{m l}}{l} \\
& =\sum_{l=1}^{\infty} \frac{1}{l} \sum_{m=1}^{\infty}\left(x^{l}\right)^{m}=\frac{x}{1-x}+\sum_{l=2}^{\infty} \frac{x^{l}}{l\left(1-x^{l}\right)}
\end{aligned}
$$

где $|x|<1$. Отсюда следует, что при $x=r_{n} e^{i \theta}$

$$
\begin{aligned}
\left|\log g\left(r_{n} e^{i \theta}\right)\right| & \leqslant \frac{r_{n}}{\left|1-r_{n} e^{i \theta}\right|}-\frac{r_{n}}{1-r_{n}}+\sum_{l=1}^{\infty} \frac{r_{n}^{l}}{l\left(1-r_{n}^{l}\right)} \\
& =r_{n}\left(\frac{1}{\left|1-r_{n} e^{i \theta}\right|}-\frac{1}{1-r_{n}}\right)+\log g\left(r_{n}\right)
\end{aligned}
$$

Теперь, используя неравенство $\left|g\left(r_{n} e^{i \theta}\right)\right| \leqslant \exp \left|\log g\left(r_{n} e^{i \theta}\right)\right|$ и лемму 3 при $\Delta_{n}=\delta_{n}$, получаем, что

$$
\begin{aligned}
\left|g\left(r_{n} e^{i \theta}\right)\right| & \leqslant g\left(r_{n}\right) \exp \left(-r_{n} 3 \sqrt{6} n^{3 / 2} \delta_{n}^{2} / \pi^{3}+O\left(\lambda^{2}\right)\right) \\
& =g\left(r_{n}\right) \exp \left(-r_{n} 3 \sqrt{6} n^{1 / 6} / \pi^{3} \log ^{2} n+O\left(\lambda^{2}\right)\right)
\end{aligned}
$$

равномерно относительно $|\theta| \geqslant \delta_{n}$.

Рассмотрим теперь отдельно два случая.

В первом случае $y=e^{w / \sigma_{n}^{\prime}(\lambda)} \geqslant 1$, то есть $w \geqslant 0$.

Заметим, что подынтегральную функцию в (33) можно представить в виде

$$
g\left(r_{n} e^{i \theta}\right) \prod_{m \geqslant s}\left(1+\frac{r_{n}^{m} e^{i \theta m}(y-1)}{1-r_{n}^{m} y}\right) e^{-i n \theta} .
$$


Оценим сверху второй множитель:

$$
\begin{aligned}
\left|\prod_{m \geqslant s}\left(1+\frac{r_{n}^{m} e^{i \theta m}(y-1)}{1-r_{n}^{m} y}\right)\right| & \leqslant \prod_{m \geqslant s}\left(1+\frac{r_{n}^{m}(y-1)}{1-r_{n}^{m} y}\right) \\
& =\exp \left\{\log \prod_{m \geqslant s}\left(1+\frac{r_{n}^{m}(y-1)}{1-r_{n}^{m} y}\right)\right\} \\
& =\exp \left\{\sum_{m \geqslant s} \log \left(1+\frac{r_{n}^{m}(y-1)}{1-r_{n}^{m} y}\right)\right\} .
\end{aligned}
$$

Внутреннюю сумму логарифмов можно оценить так же, как это было сделано в разделе 2 при выводе (20). Таким образом, с учетом (6), (38) и (41) при фиксированном $w \geqslant 0$ получаем, что

$$
\begin{aligned}
\sum_{m \geqslant s} \log \left(1+\frac{(y-1) r_{n}^{m}}{1-r_{n}^{m} y}\right) & \sim-\frac{1}{\log r_{n}} \int_{\lambda}^{\infty} \log \left(1+\frac{e^{-u}(y-1)}{1-e^{-u} y}\right) d u \\
& \leqslant \frac{(6 n)^{1 / 2}(y-1)}{\pi} \int_{\lambda}^{\infty} \frac{e^{-u}}{1-e^{-u} y} d u \\
& =-\frac{(6 n)^{1 / 2} w}{\sigma_{n}^{\prime}(\lambda) \pi y} \log \left(1-e^{-\lambda} y\right)+O(1)
\end{aligned}
$$

Собирая вместе (2), (38) и (54)-(57), получаем, что для достаточно больших $n$ и некоторого $\varepsilon>0$

$$
\begin{aligned}
\left|J_{2}(y)\right| e^{-\mu_{n}(\lambda) w / \sigma_{n}^{\prime}(\lambda)} \leqslant & \frac{e^{-\mu_{n}(\lambda) w / \sigma_{n}^{\prime}(\lambda)}}{2 \pi} \int_{\delta_{n} \leqslant|\theta| \leqslant \pi}\left|g\left(r_{n} e^{i \theta}\right)\right| \prod_{m \geqslant s}\left|1+\frac{r_{n}^{m} e^{i \theta m}(y-1)}{1-r_{n}^{m} y}\right| d \theta \\
& \leqslant g\left(r_{n}\right) \exp \left\{-r_{n} 3 \sqrt{6} n^{1 / 6} / \pi^{3} \log ^{2} n+O\left(\lambda^{2}\right)\right. \\
& \left.\quad-\frac{(6 n)^{1 / 2} w}{\sigma_{n}^{\prime}(\lambda) \pi y} \log \left(1-e^{-\lambda} y\right)+\frac{(6 n)^{1 / 2} w}{\pi \sigma_{n}^{\prime}(\lambda)} \log \left(1-e^{-\lambda}\right)\right\} \\
= & O\left(g\left(r_{n}\right) \exp \left\{-(1-\varepsilon) 3 \sqrt{6} n^{1 / 6} /\left(\pi^{3} \log ^{2} n\right)+O\left(\lambda^{2}\right)\right\}\right) .
\end{aligned}
$$

Кроме того, в силу (14)

$$
\log b^{1 / 2}\left(r_{n}\right)=\frac{3}{4} \log n+O(1) .
$$

Отсюда и из первого условия теоремы 4 следует, что при $n \rightarrow \infty$

$$
\begin{aligned}
& g\left(r_{n}\right) \exp \left\{-(1-\varepsilon) 3 \sqrt{6} n^{1 / 6} /\left(\pi^{3} \log ^{2} n\right)+O\left(\lambda^{2}\right)\right\} \\
& \quad=b^{-1 / 2}\left(r_{n}\right) g\left(r_{n}\right) \exp \left\{-(1-\varepsilon) 3 \sqrt{6} n^{1 / 6} /\left(\pi^{3} \log ^{2} n\right)+O\left(\lambda^{2}\right)+\frac{3}{4} \log n\right\} \\
& \quad=b^{-1 / 2}\left(r_{n}\right) g\left(r_{n}\right) \exp \left\{-(1-\varepsilon) 3 \sqrt{6} n^{1 / 6} /\left(\pi^{3} \log ^{2} n\right)+O\left(\log ^{2} n\right)\right\} \\
& =o\left(g\left(r_{n}\right) / b^{1 / 2}\left(r_{n}\right)\right) .
\end{aligned}
$$


Теперь из (58) следует, что

$$
\left|J_{2}(y)\right| e^{-\mu_{n}(\lambda) w / \sigma_{n}^{\prime}(\lambda)}=o\left(g\left(r_{n}\right) / b^{1 / 2}\left(r_{n}\right)\right) .
$$

Во втором случае $y=e^{w / \sigma_{n}^{\prime}(\lambda)}<1$, то есть $w<0$.

Для того чтобы оценить произведение в подынтегрпльной функции в (55) с множителем $e^{-\mu_{n}(\lambda) w / \sigma_{n}^{\prime}(\lambda)}$, прежде всего используем равенство $\left|e^{\log z}\right|=e^{\log |z|}$. Тогда

$$
\begin{aligned}
e^{-\mu_{n}(\lambda) w / \sigma_{n}^{\prime}(\lambda)}\left|\exp \left\{\log \prod_{m \geqslant s}\left(1+\frac{r_{n}^{m} e^{i \theta m}(y-1)}{1-r_{n}^{m} e^{i \theta m}}\right)\right\}\right| \\
=e^{-\mu_{n}(\lambda) w / \sigma_{n}^{\prime}(\lambda)} \\
\times \exp \left\{\sum_{m \geqslant s} \log \left(1-\frac{(1-y) r_{n}^{m}}{1-r_{n}^{m}}\right)\right\} \\
\times \exp \left\{-\sum_{m \geqslant s} \log \left(1-\frac{(1-y) r_{n}^{m}}{1-r_{n}^{m}}\right)\right\} \\
\times \exp \left\{\sum_{m \geqslant s} \log \left|1-\frac{(1-y) r_{n}^{m} e^{i \theta m}}{1-r_{n}^{m} e^{i \theta m}}\right|\right\} .
\end{aligned}
$$

Теперь ясно, что в силу (38), (20), (41) и (2),

$$
\begin{aligned}
\sum_{m \geqslant s} \log \left(1-\frac{(1-y) r_{n}^{m}}{1-r_{n}^{m}}\right) & \leqslant-(1-y) \sum_{m \geqslant s} \frac{r_{n}^{m}}{1-r_{n}^{m}} \\
& =\left(w / \sigma_{n}^{\prime}(\lambda)+O\left(\left(\sigma_{n}^{\prime}\right)^{-2}(\lambda)\right)\right) d_{1}\left(r_{n}\right) \\
& =-\frac{w(6 n)^{1 / 2}}{\sigma_{n}^{\prime}(\lambda) \pi} \log \left(1-e^{-\lambda}\right)+O(1)=\mu_{n}(\lambda) w / \sigma_{n}^{\prime}(\lambda)+O(1) .
\end{aligned}
$$

Таким образом, мы получили оценку

$$
\begin{aligned}
e^{-\mu_{n}(\lambda) w / \sigma_{n}^{\prime}(\lambda)} \exp \left\{\sum_{m \geqslant s} \log \left(1-\frac{(1-y) r_{n}^{m}}{1-r_{n}^{m}}\right)\right\} \\
=e^{-\mu_{n}(\lambda) w / \sigma_{n}^{\prime}(\lambda)} \exp \left\{\mu_{n}(\lambda) w / \sigma_{n}^{\prime}(\lambda)+O(1)\right\}=O(1) .
\end{aligned}
$$

Оценим теперь оставшийся множитель в (60). Заметим, что

$$
\begin{aligned}
\sum_{m \geqslant s} & \left(\log \left|1-\frac{(1-y) r_{n}^{m} e^{i \theta m}}{1-r_{n}^{m} e^{i \theta m}}\right|-\log \left(1-\frac{(1-y) r_{n}^{m}}{1-r_{n}^{m}}\right)\right) \\
& =\sum_{m \geqslant s}\left(\frac{1}{2} \log \frac{1-2(2-y) r_{n}^{m} \cos m \theta+(2-y)^{2} r_{n}^{2 m}}{1-2 r_{n}^{m} \cos m \theta+r_{n}^{2 m}}-\log \frac{1-(2-y) r_{n}^{m}}{1-r_{n}^{m}}\right) .
\end{aligned}
$$

Детали докаӟательства этой формулы, которые заключаются в простых алгебраических преобразований комплексных чисел, мы оставляем читателю. Далее, прямые 
вычисления приводят к следующей оценке аргумента первого логарифма в (62):

$$
\begin{aligned}
& \frac{1-2(2-y) r_{n} \cos m \theta+(2-y)^{2} r_{n}^{2 m}}{1-2 r_{n}^{m} \cos m \theta+r_{n}^{2 m}} \\
& =1-2(1-y) \frac{r_{n}^{m} \cos m \theta}{1-2 r_{n}^{m} \cos m \theta+r_{n}^{2 m}}+2(1-y) \frac{r_{n}^{2 m}}{1-2 r_{n}^{m} \cos m \theta+r_{n}^{2 m}} \\
& \quad+\frac{(1-y)^{2} r_{n}^{2 m}}{1-2 r_{n}^{m} \cos m \theta+r_{n}^{2 m}} \\
& \leqslant 1-2(1-y) \frac{r_{n}^{m}(\cos m \theta-1+1)}{1-2 r_{n}^{m} \cos m \theta+r_{n}^{2 m}}+2(1-y) \frac{r_{n}^{2 m}}{1-2 r_{n}^{m} \cos m \theta+r_{n}^{2 m}}+\frac{(1-y)^{2} r_{n}^{2 m}}{\left(1-r_{n}^{m}\right)^{2}} \\
& \leqslant 1+2(1-y) \frac{r_{n}^{m}(1-\cos m \theta)}{\left(1-r_{n}^{m}\right)^{2}}-2(1-y) \frac{r_{n}^{m}\left(1-r_{n}^{m}\right)}{1-2 r_{n}^{m} \cos m \theta+r_{n}^{m}}+\frac{(1-y)^{2} r_{n}^{2 m}}{\left(1-r_{n}^{m}\right)^{2}} .
\end{aligned}
$$

Рассмотрим теперь аргумент второго логарифма в (62):

$$
\frac{1-(2-y) r_{n}^{m}}{1-r_{n}^{m}}=1-\frac{(1-y) r_{n}^{m}}{1-r_{n}^{m}}
$$

Учитывая (63) и (64), получаем, что слагаемое правой суммы в (62) не превосходит

$$
\begin{aligned}
(1-y) r_{n}^{m}( & \left.\frac{1}{1-r_{n}^{m}}-\frac{1-r_{n}^{m}}{1-2 r_{n}^{m} \cos m \theta+r_{n}^{2 m}}\right) \\
& \quad+(1-y) \frac{r_{n}^{m}(1-\cos m \theta)}{\left(1-r_{n}^{m}\right)^{2}}+O\left((1-y)^{2} r_{n}^{2 m} /\left(1-r_{n}^{m}\right)^{2}\right) \\
= & (1-y) \frac{2 r_{n}^{m}(1-\cos m \theta)}{\left(1-r_{n}^{m}\right)\left(1-2 r_{n}^{m} \cos m \theta+r_{n}^{2 m}\right)} \\
& \quad+(1-y) \frac{r_{n}^{m}(1-\cos m \theta)}{\left(1-r_{n}^{m}\right)^{2}}+O\left((1-y)^{2} r_{n}^{2 m} /\left(1-r_{n}^{m}\right)^{2}\right) \\
\leqslant & (1-y)(1-\cos m \theta)\left(\frac{2 r_{n}^{2 m}}{\left(1-r_{n}^{m}\right)^{3}}+\frac{r_{n}^{m}}{\left(1-r_{n}^{m}\right)^{2}}\right) \\
& \quad+O\left((1-y)^{2} r_{n}^{2 m} /\left(1-r_{n}^{m}\right)^{2}\right) \\
= & (1-y)(1-\cos m \theta) \frac{r_{n}^{m}\left(1+r_{n}^{m}\right)}{\left(1-r_{n}^{m}\right)^{3}}+O\left((1-y)^{2} r_{n}^{2 m} /\left(1-r_{n}^{m}\right)^{2}\right) \\
< & \frac{2(1-y)}{\left(1-r_{n}^{s}\right)^{3}}(1-\cos m \theta) r_{n}^{m}+O\left((1-y)^{2} r_{n}^{2 m} /\left(1-r_{n}^{m}\right)^{2}\right) .
\end{aligned}
$$

Оценка сверху для $r_{n}^{s}$ получается из (6) и предположения теоремы 4 относительно параметра $s$ :

$$
r_{n}^{s}=\exp \left\{\lambda \frac{(6 n)^{1 / 2}}{\pi} \log \left(1-\pi /(6 n)^{1 / 2}-\lambda /(2 n)+O\left(\lambda^{2} / n^{3 / 2}\right)\right)\right\} \leqslant e^{-\lambda}
$$


Подставляя (66) и (38) в (65) и затем (65) в (62), получаем, что

$$
\begin{aligned}
S_{s, n}= & \sum_{(m \geqslant s}\left(\log \left|1-\frac{(1-y) r_{n}^{m} e^{i \theta m}}{1-r_{n}^{m} e^{i \theta m}}\right|-\log \left(1-\frac{(1-y) r_{n}^{m}}{1-r_{n}^{m}}\right)\right) \\
\leqslant & -\frac{2 w}{\left(1-e^{-\lambda}\right)^{3} \sigma_{n}^{\prime}(\lambda)}\left(1+O\left(1 / \sigma_{n}^{\prime}(\lambda)\right)\right) \\
& \times \sum_{m \geqslant s}\left((1-\cos m \theta) r_{n}^{m}+O\left(r_{n}^{2 m} /\left(\sigma_{n}^{\prime}(\lambda)\left(1-r_{n}^{m}\right)^{2}\right)\right)\right) \\
= & -\frac{2 w}{\left(1-e^{-\lambda}\right)^{3} \sigma_{n}^{\prime}(\lambda)}\left(\frac{r_{n}^{s}}{1-r_{n}}-\Re\left(\frac{r_{n}^{s} e^{i \theta s}}{1-r_{n} e^{i \theta}}\right)\right)+O\left(d_{2}\left(r_{n}\right) /{\sigma_{n}^{\prime}}^{2}(\lambda)\right) .
\end{aligned}
$$

Теперь в силу (23) и (4)

$$
S_{s, n}=-\frac{2 w r_{n}^{s}}{\left(1-e^{-\lambda}\right)^{3} \sigma_{n}^{\prime}(\lambda)}\left(\frac{1}{1-r_{n}}-\Re\left(\frac{e^{i \theta s}}{1-r_{n} e^{i \theta}}\right)\right)+O(1)
$$

и, наконец, в силу (66)

$$
\begin{aligned}
S_{s, n}=- & \frac{2 w e^{-\lambda}}{\left(1-e^{-\lambda}\right)^{3} \sigma_{n}^{\prime}(\lambda)}\left(\frac{1}{1-r_{n}}-\frac{\cos \left(\theta s-\arg \left(1-r_{n} e^{i \theta}\right)\right)}{\left|1-r_{n} e^{i \theta}\right|}\right)+O(1) \\
=- & \frac{2 w e^{-\lambda}}{\left(1-e^{-\lambda}\right)^{3} \sigma_{n}^{\prime}(\lambda)}\left(\frac{1}{1-r_{n}}-\frac{1}{\left|1-r_{n} e^{i \theta}\right|}\right) \\
& +\frac{2 w e^{-\lambda}\left(\cos \left(\theta s-\arg \left(1-r_{n} e^{i \theta}\right)\right)-1\right)}{\left(1-e^{-\lambda}\right)^{3} \sigma_{n}^{\prime}(\lambda)\left|1-r_{n} e^{i \theta}\right|}+O(1) .
\end{aligned}
$$

Для получения требуемой оценки интеграла $J_{2}(y)$, определенного равенствами (33) и (55), воспользуемся полученной выше оценкой и оценками (52), (61). Оценки, полученные к настоящему моменту во втором случае, можно записать следующим образом:

$$
\begin{aligned}
& e^{-\mu_{n}(\lambda) w / \sigma_{n}^{\prime}(\lambda)}\left|J_{2}(y)\right| \leqslant \frac{1}{2 \pi} e^{-\mu_{n}(\lambda) w / \sigma_{n}^{\prime}(\lambda)} \int_{\delta_{n} \leqslant|\theta| \leqslant \pi}\left|g\left(r_{n} e^{i \theta}\right)\right| \\
& \times \exp \left\{\sum_{m \geqslant s} \log \left(1-\frac{(1-y) r_{n}^{m}}{1-r_{n}^{m}}\right)\right\} \\
& \times \exp \left\{-\sum_{m \geqslant s} \log \left(1-\frac{(1-y) r_{n}^{m}}{1-r_{n}^{m}}\right)\right\} \\
& \times \exp \left\{\sum_{m \geqslant s} \log \left|1-\frac{(1-y) r_{n}^{m} e^{i \theta m}}{1-r_{n}^{m} e^{i \theta m}}\right|\right\} d \theta \\
& \leqslant \frac{g\left(r_{n}\right)}{2 \pi} \exp \left\{-w q(\theta) / \sigma_{n}^{\prime}(\lambda)+O(1)\right\} \\
& \times \int_{\delta_{n} \leqslant|\theta| \leqslant \pi} \exp \left\{\left(r_{n}+\frac{2 w e^{-\lambda}}{\left(1-e^{-\lambda}\right)^{3} \sigma_{n}^{\prime}(\lambda)}\right)\right. \\
&\left.\times\left(\frac{1}{1-r_{n}}-\frac{1}{\mid 1-r_{n} e^{i \theta \mid}}\right)+O(1)\right\} d \theta
\end{aligned}
$$


где

$$
q(\theta)=\frac{2 e^{-\lambda}\left(1-\cos \left(\theta s-\arg \left(1-r_{n} e^{i \theta}\right)\right)\right)}{\left(1-e^{-\lambda}\right)^{3}\left|1-r_{n} e^{i \theta}\right|}
$$

Используя оценку снизу для $\lambda$ из условия теоремы 4, и соотношения (6) и (41), получаем, что

$$
r_{n}+\frac{2 w e^{-\lambda}}{\left(1-e^{-\lambda}\right)^{3} \sigma_{n}^{\prime}(\lambda)} \geqslant 1-\varepsilon
$$

при некотором $\varepsilon>0$ и произвольном фиксированном $w<0$. Поэтому (67) можно переписать в виде

$$
e^{-\mu_{n}(\lambda) w / \sigma_{n}^{\prime}(\lambda)}\left|J_{2}(y)\right|=O\left(g\left(r_{n}\right) I_{n}\right)
$$

где

$$
\begin{aligned}
I_{n}=\int_{\delta_{n} \leqslant|\theta| \leqslant \pi} \exp \left\{(1-\varepsilon)\left(\frac{1}{\mid 1-r_{n} e^{i \theta \mid}}-\frac{1}{1-r_{n}}\right)+O(1)\right\} & \\
& \quad \times \exp \left\{-w q(\theta) / \sigma_{n}^{\prime}(\lambda)+O(1)\right\} d \theta
\end{aligned}
$$

и $q(\theta)$ определено в (68). Чтобы закончить доказательство, то есть получить (59), остается показать, что

$$
I_{n}=o\left(b^{-1 / 2}\left(r_{n}\right)\right)
$$

Представим $I_{n}$ в виде суммы

$$
I_{n}=I_{n}^{\prime}+I_{n}^{\prime \prime}
$$

где

$$
\begin{aligned}
I_{n}^{\prime}= & \int_{\delta_{n} \leqslant|\theta|<(\log n) / n^{7 / 12}} \exp \left\{(1-\varepsilon)\left(\frac{1}{\left|1-r_{n} e^{i \theta}\right|}-\frac{1}{1-r_{n}}\right)+O(1)\right\} \\
& \times \exp \left\{-w q(\theta) / \sigma_{n}^{\prime}(\lambda)+O(1)\right\} d \theta \\
I_{n}^{\prime \prime}= & \int_{(\log n) / n^{7 / 12} \leqslant|\theta| \leqslant \pi} \exp \left\{(1-\varepsilon)\left(\frac{1}{\mid 1-r_{n} e^{i \theta \mid}}-\frac{1}{1-r_{n}}\right)+O(1)\right\} \\
& \quad \times \exp \left\{-w q(\theta) / \sigma_{n}^{\prime}(\lambda)+O(1)\right\} d \theta .
\end{aligned}
$$

Далее $c_{0}, c_{1}, \ldots$ обозначают явно вычисляемые положительные постоянные.

Чтобы оценить $I_{n}^{\prime}$, заметим, что из (6) следует оценка

$$
\begin{aligned}
\frac{1}{\mid 1-r_{n} e^{i \theta \mid}} & =\left(1+r_{n}^{2}-2 r_{n} \cos \theta\right)^{-1 / 2} \\
& =\left(\pi^{2} / 6 n+\theta^{2}-\theta^{2} \pi /(6 n)^{1 / 2}+O\left(\theta^{2} / n\right)+\theta^{4}\right)^{-1 / 2} \leqslant(6 n)^{1 / 2} / \pi
\end{aligned}
$$

С другой стороны, при $\delta_{n} \leqslant|\theta|<(\log n) / n^{7 / 12}$

$$
1-\cos \left(\theta s-\arg \left(1-r_{n} e^{i \theta}\right)\right) \leqslant\left(\theta s-\arg \left(1-r_{n} e^{i \theta}\right)\right)^{2} / 2 \leqslant c_{0} n \theta^{2}
$$


в силу условия теоремы 4 относительно $s$. Поэтому согласно (41)

$$
-\frac{w q(\theta)}{\sigma_{n}^{\prime}(\lambda)} \leqslant c_{1}(-w) n^{5 / 4} \theta^{2}
$$

для всех $\lambda$, удовлетворяющих первому и второму условиям теоремы 4. Применяя это неравенство и лемму 3 при $\Delta_{n}=\delta_{n}$ для оценки подынтегральной функции в $I_{n}^{\prime}$, находим, что при $w<0$ для некоторого $\eta \in\left(0,(1-\varepsilon) 3 \sqrt{6} / \pi^{3}\right)$

$$
\begin{aligned}
I_{n}^{\prime} & \leqslant 2 \int_{\delta_{n} \leqslant \theta<(\log n) / n^{7 / 12}} \exp \left\{c_{2}(-w) n^{5 / 4} \theta^{2}\right\} \exp \left\{-(1-\varepsilon) 3 \sqrt{6} n^{3 / 2} \delta_{n}^{2} / \pi^{3}\right\} d \theta \\
& =O\left(\frac{\log n}{n^{7 / 12}} \exp \left\{c_{2}(-w) n^{5 / 4}\left(\log ^{2} n\right) n^{-7 / 6}-(1-\varepsilon) 3 \sqrt{6} n^{1 / 6} /\left(\pi^{3} \log ^{2} n\right)\right\}\right) \\
& =O\left(\frac{\log n}{n^{7 / 12}} \exp \left\{n^{1 / 6} /\left(\log ^{2} n\right)\left(c_{1}(-w) n^{-1 / 12} \log ^{4} n-(1-\varepsilon) 3 \sqrt{6} / \pi^{3}\right)\right\}\right) \\
& =O\left(\frac{\log n}{n^{7 / 12}} \exp \left\{n^{1 / 6} /\left(\log ^{2} n\right)\left(\eta-(1-\varepsilon) 3 \sqrt{6} / \pi^{3}\right)\right\}\right) .
\end{aligned}
$$

Наконец, для оценки $I_{n}^{\prime \prime}$ заметим, что из (73) следует, что

$$
\frac{1}{\mid 1-r_{n} e^{i \theta \mid}} \leqslant \frac{1}{|\theta|}
$$

Подставляя эту оценку в (68) и используя (41), находим, что

$$
\frac{(-w) q(\theta)}{\sigma_{n}^{\prime}(\lambda)} \leqslant c_{2}(-w) n^{-1 / 4} /|\theta|
$$

Теперь, применяя так же, как и выше, лемму 3 при $\Delta_{n}=(\log n) / n^{7 / 12}$, находим, что при $w<0$

$$
\begin{aligned}
I_{n}^{\prime \prime} & =O\left(\exp \left\{\left(n^{1 / 3} \log ^{2} n\right)\left(c_{2}(-w) / \log ^{3} n-(1-\varepsilon) 3 \sqrt{6} / \pi^{3}\right)\right\}\right) \\
& =O\left(\exp \left\{\left(n^{1 / 3} \log ^{2} n\right)\left(\eta-(1-\varepsilon) 3 \sqrt{6} / \pi^{3}\right)\right\}\right) .
\end{aligned}
$$

Применяя (72), (74), (75) и аргументы, использованные для оценки $b^{1 / 2}\left(r_{n}\right)$ в первом случае, получаем соотношение (71), которое с учетом (69) приводит к (59). Теперь, применяя лемму 4 , находим, что при $n \rightarrow \infty$

$$
J_{2}\left(e^{w / \sigma_{n}^{\prime}(\lambda)}\right) \frac{e^{-\mu_{n}(\lambda) w / \sigma_{n}^{\prime}(\lambda)}}{p_{n} r_{n}^{n}}=o(1) .
$$

Наконец, применяя модификацию Куртисса теоремы Леви о непрерывном соответствии между распределениями и характеристическими функциями, из соотношений $(31),(51)$ и (76) заключаем, что распределдение случайной величины

$$
\left(L_{s, n}-\mu_{n}(\lambda)\right) / \sigma_{n}^{\prime}(\lambda)
$$

слабо сходится к нормальному распределению с нулевым средним и дисперсией 1 $C^{2}(\lambda)$. С помощью перенормировки (37) получаем требуемый результат.

Автор благодарен рецензенту за полезные замечания. 


\section{Список литературы}

1. Abramovitz M., Stegun I. A. Handbook of Mathematical Functions with Formulas, Graphs and Mathematical Tables. Dover Publ., New York, 1965.

2. Andrews G. E. The Theory of Partitions. Addison-Wesley, Reading, Mass., 1976.

3. Arratia R., Tavaré S. Independent process approximations for random combinatorial structures. Adv. Math. (1994) 104, 90-154.

4. Auluck F. C., Chowla S., Gupta H. On the maximum of the number of partitions of $n$ into $k$ parts. J. Indian Math. Soc. (1942) 6, 105-112.

5. Curtiss J. A note on the theory of moment generating functions. Ann. Math. Statist. (1942) 13, 430-433.

6. Erdös P., Lehner J. The distribution of the number of summands in the partitions of a positive integers. Duke Math. J. (1941) 8, 335-345.

7. Fristedt B. The structure of random partitions of large integers. Trans. Amer. Math. Soc. (1993) 337, 703-735.

8. Hardy G. H., Ramanujan S. Asymptotic formulae in combinatory analysis. Proc. London Math. Soc. (1918) 17, 75-115.

9. Hayman W. K. A generalization of Stirling's formula, J. Reine Angew. Math. (1956) 196, 67-95.

10. Hwang H. K. Distribution of integer partitions with large number of summands. Acta Arithm. (1997) 78, 351-365.

11. Колчин В. Ф. Случайнъе отображения. Наука, Москва, 1984.

12. Odlyzko A. M., Richmond L. B. Asymptotic expansions for the coefficients of analytic generating functions. Aequationes Math. (1985) 28, 50-63.

13. Pittel B. On a likely shape of the random Ferrers diagram. Adv. Appl. Math. (1997) 18, 432-488.

14. Постников А. Г. Введение в аналитическую теорию чисел. Наука, Москва, 1971.

15. Stromberg K. R. Introduction to Classical Real Analysis. Wadsworth, New York, 1981.

16. Szalay M., Turán P. On some problems of the statistical theory of partitions with application to characters of the symmetric group I. Acta Math. Acad. Sci. Hungar. (1977) 29, 361-379.

17. Szalay M., Turán P. On some problems of the statistical theory of partitions with application to characters of the symmetric group II. Acta Math. Acad. Sci. Hungar. (1977) 29, 381-392.

18. Szalay M., Turán P. On some problems of the statistical theory of partitions with application to characters of the symmetric group III, Acta Math. Acad. Sci. Hungar. (1978) 32, 129-155.

Статья поступила 19.09.1998. 